\title{
Acquired cystic disease-associated renal tumors: an immunohistochemical and fluorescence in situ hybridization study
}

\author{
Paolo Cossu-Rocca ${ }^{1,2}$, John N Eble ${ }^{1}$, Shaobo Zhang ${ }^{1}$, Guido Martignoni ${ }^{3}$, Matteo Brunelli ${ }^{3}$ \\ and Liang Cheng ${ }^{1}$ \\ ${ }^{1}$ Departments of Pathology and Laboratory Medicine, Indiana University, Indianapolis, IN, USA; \\ ${ }^{2}$ Dipartimento di Patologia, Universitá di Sassari, Sassari, Italy and ${ }^{3}$ Dipartimento di Patologia, \\ Universitá di Verona, Verona, Italy
}

\begin{abstract}
End-stage renal disease is associated with an increased incidence of renal cell neoplasms. Among these, recent studies have identified tumors with unusual histological patterns that do not fit into the categories recognized in the current classification system. These tumors often occur in kidneys with acquired cystic disease and are composed mainly of large eosinophilic cells arranged in solid, cribriform, acinar, or papillary patterns. They also contain deposits of calcium oxalate crystals. We investigated three eosinophilic epithelial tumors arising in kidneys with acquired cystic disease from three patients. Each of the tumors was composed of large eosinophilic cells arranged in solid, acinar, or tubulocystic architecture. Deposits of calcium oxalate crystals were present in each tumor. Hale's colloidal stain showed a positive cytoplasmic reaction in one of the neoplasms. Immunohistochemistry displayed positive results for CD10 (3/3), AE1/AE3 (3/3), alpha-methylacylCoA racemase (2/3), CAM5.2 (2/3), and vimentin (1/3). Reactions for epithelial membrane antigen, cytokeratin 7, and high molecular weight cytokeratin $(34 \beta \mathrm{E} 12)$ were negative. Fluorescence in situ hybridization analysis showed no losses or gains of chromosomes 1, 2, 6, 10, or 17 in one tumor. There were gains of chromosomes 1 , 2 , and 6 in two tumors. One of these tumors also showed gains of chromosome 10. Eosinophilic renal cell tumors associated with acquired cystic disease have immunophenotypes and genetic profiles distinct from the renal cell neoplasms recognized in the current classification of renal cell neoplasia, and should be considered as a distinct clinicopathologic entity in the spectrum of renal cell neoplasia.

Modern Pathology (2006) 19, 780-787. doi:10.1038/modpathol.3800604; published online 31 March 2006
\end{abstract}

Keywords: kidney tumors; end-stage renal disease; acquired cystic disease of kidney; calcium oxalate; cytogenetics; fluorescence in situ hybridization (FISH)

End-stage renal disease is known to be associated with an increased risk of developing renal cell neoplasms, particularly in patients with acquired cystic kidney disease secondary to long-term hemodialysis. ${ }^{1-3}$ Clinically, these tumors differ from the sporadic variants with a younger age of presentation, greater frequency of multicentricity and bilaterality, and possibly an overall better prognosis. ${ }^{1-7}$

Different types of renal cell epithelial neoplasms have been described in association with end-stage renal disease, with a high prevalence of papillary

Correspondence: Dr L Cheng, MD, Departments of Pathology and Laboratory Medicine, Indiana University Medical Center, University Hospital 3465, 550 North University Boulevard, Indianapolis, IN 46202, USA.

E-mail: lcheng@iupui.edu

Received 9 January 2006; revised 6 March 2006; accepted 10 March 2006; published online 31 March 2006 renal cell carcinomas and clear-cell renal carcinomas. ${ }^{1,3,4,7-12}$ However, tumors arising in end-stage renal diseases, especially in association with acquired cystic disease, may also show distinctive histologic features not easily referable to the categories described in the current WHO classification system. ${ }^{13-15}$ These tumors are characterized by abundant eosinophilic cytoplasm, a variably solid, cribriform, tubulo-cystic and papillary architecture, and by deposits of calcium oxalate crystals. ${ }^{13,14}$

Little is known about the immunohistochemical phenotype and genetic profiles of these tumors. In this study, we analyzed three such tumors occurring in patients with acquired cystic disease. We investigated the expression of cytokeratins, vimentin, CD10, and alpha-methylacyl-CoA racemase (AMACR) using immunohistochemistry. We also studied, by fluorescence in situ hybridization (FISH), the complement of chromosomes 1, 2, 6, 10, 
and 17, which are known to be frequently lost in eosinophilic chromophobe renal cell carcinoma; chromosome 1 is lost in approximately $10 \%$ of renal oncocytomas. ${ }^{16-20}$

\section{Materials and methods}

\section{Patients}

Three renal epithelial tumors from three patients with end-stage renal disease and acquired cystic disease were retrieved from the archives of the Department of Pathology of the Indiana University School of Medicine. From paraffin blocks representative of the tumors and adjacent non-neoplastic renal parenchyma, 4- $\mu$ m-thick sections were cut and stained with hematoxylin and eosin and Hale's colloidal iron stain. Additional consecutive slides were utilized for immunohistochemical staining and FISH analysis.

\section{Immunohistochemical Staining}

Immunohistochemistry was performed with the following antibodies: vimentin (DAKO, Carpinteria, CA, USA; clone V9; prediluted); CAM5.2 (Becton Dickinson, Mountain View, CA, USA; prediluted); AE1/AE3 (DAKO; Clone AE1/AE3; 1:80 dilution), epithelial membrane antigen (EMA) (DAKO; clone E29; prediluted); cytokeratin 7 (CK7) (DAKO; clone OV-TL 12/30; prediluted); high molecular weight keratin (HMWK) (DAKO; clone 34 $\beta$ E12; prediluted); CD10 (Cell Marque, Hot Springs, AR, USA; clone 56C6; prediluted); AMACR (DAKO; clone P504S; 1:100 dilution).

Briefly, slides were deparaffinized twice in xylene for $5 \mathrm{~min}$ and rehydrated through graded ethanol solutions to distilled water. Antigen retrieval was performed by heating sections in citrate buffer (AE1/AE3, CD10, and AMACR) or enzymatically with proteinase K (CAM5.2 and CK7) and pepsin (HMWK). No pretreatment was utilized for vimentin and EMA immunostaining. Inactivation of endogenous peroxidase activity was obtained by incubating sections in $3 \% \mathrm{H}_{2} \mathrm{O}_{2}$ for $15 \mathrm{~min}$. Localization of bound antibodies was performed with peroxidaselabeled streptavidin-biotin system (DAKO, LSAB2 Kit) with $3,3^{\prime}$-diaminobenzidine as a chromogen. Appropriate positive controls for each antibody were run concurrently and showed adequate immunostaining.

\section{Fluorescence In Situ Hybridization}

The slides were deparaffinized with two washes of xylene, $15 \mathrm{~min}$ each, and subsequently washed twice with absolute ethanol, 10 min each and then air dried in the hood. Next, the slides were treated with $0.1 \mathrm{mM}$ citric acid (pH 6.0) (Zymed, CA, USA) at $95^{\circ} \mathrm{C}$ for $10 \mathrm{~min}$, rinsed in distilled water for $3 \mathrm{~min}$ followed by a wash of $2 \times$ SSC (standard saline citrate) for $5 \mathrm{~min}$. Digestion of the tissue was performed by applying $0.4 \mathrm{ml}$ of pepsin $(5 \mathrm{mg} / \mathrm{ml}$ in $0.9 \% \mathrm{NaCl}, \mathrm{pH} 1.5$ ) (Sigma, St Louis, MO, USA) at $37^{\circ} \mathrm{C}$ for $40 \mathrm{~min}$. The slides were rinsed with distilled water for $3 \mathrm{~min}$, then washed with $2 \times$ SSC for $5 \mathrm{~min}$ and air dried. FISH was performed with centromeric $\alpha$-satellite DNA probes for chromosome 1 (Centromeric Enumeration Probe, CEP 1, Spectrum Orange), chromosome 2 (CEP2, Spectrum Orange), chromosome 6 (CEP 6, Spectrum Green), chromosome 10 (CEP 10, Spectrum Green), and chromosome 17 (CEP 17, Spectrum Orange). All of the probes were from Vysis (Downers Grove, IL, USA) and were diluted with tDenHyb1 (Insitus, Alburquerque, NM, USA) in a ratio of 1:100. A $5 \mu$ l portion of diluted probe was applied to each slide in reduced light. The slides were then covered with a $22 \times 22 \mathrm{~mm}$ coverslip and sealed with rubber cement. Denaturation was achieved by incubating the slides at $80^{\circ} \mathrm{C}$ for $10 \mathrm{~min}$ in a humidified box and then at $37^{\circ} \mathrm{C}$ overnight. The coverslips were removed and the slides were washed with two washes at $45^{\circ} \mathrm{C}$ with $0.1 \mathrm{XSSC} / 1.5 \mathrm{M}$ urea $(20 \mathrm{~min}$ for each wash), followed by a wash with 2XSSC for $20 \mathrm{~min}$ and a wash with $2 \mathrm{XSSC} / 0.1 \% \mathrm{NP}-40$ for $10 \mathrm{~min}$ at $45^{\circ} \mathrm{C}$. The slides were further washed with room temperature 2XSSC for $5 \mathrm{~min}$. The slides were air dried and counterstained with $10 \mu \mathrm{l}$ DAPI (Insitus, Albuquerque, NM, USA), covered with coverslips and sealed with nail polish. ${ }^{21-23}$

The slides were examined using a Zeiss Axioplan 2 microscope (ZEISS, Göttingen, Germany) with the following filters: SP-100 DAPI, FITC MF-101 for Spectrum Green (CEP 6 and 10) and Gold 31003 for Spectrum Orange (CEP 1, 2, and 17) from Chroma (Chroma, Brattleboro, VT, USA). The images were acquired with a CCD camera and analyzed with MetaSystem Isis Software (MetaSystem, Belmont, MA, USA). Five sequential focus stacks with $0.4 \mu \mathrm{m}$ intervals were acquired and then integrated into a single image in order to reduce thickness-related artifacts.

\section{In Situ Hybridization Analysis}

The method of analysis was described previously. ${ }^{16,24,25}$ In brief, for each slide, from 100 to 150 nuclei were scored for signals from centromeric probes under the fluorescence microscope with $\times 1000$ magnification in both tumors and nonneoplastic kidney parenchyma. Definition of chromosomal gain was based on the Gaussian model and related to the non-neoplastic controls. The cutoff values for each probe were set at mean values plus three standard deviations (s.d.) of the percentages of signals in control specimens. Any tumor with percentages of signals beyond the cutoff values was considered to have gain or loss of specific chromosomes. 
The distribution of signals was assessed on renal normal parenchyma adjacent to the tumors. For chromosomes 1, 2, 6, 10, and 17, the mean percentages of nuclei with two hybridization signals were, respectively, 59.4, 58.9, 62.3, 62.3, and 59.5\%. The mean percentages of nuclei with single signals were, respectively, 37.4, 38, 35, 35.3, and $38 \%$. The mean percentages of nuclei with three or more signals were, respectively, 3.2, 3.1, 2.8, 2.4, and $2.5 \%$. The s.d. of the mean numbers of nuclei with single signals for chromosomes $1,2,6,10$, and 17 were, respectively, $1.7,1.1,1.1,1.3$, and $0.9 \%$. The s.d. of the mean numbers of nuclei with three or more signals for chromosomes $1,2,6,10$, and 17 were, respectively, $0.8,0.4,0.5,1$, and $0.5 \%$. Therefore, the cutoff values to determine chromosomal losses or gains were, respectively, $42.5 \%$ for nuclei with single signals and $5.6 \%$ for nuclei with three or more signals for chromosome 1; 41.3 and $4.3 \%$ for chromosome 2; 38.3 and $4.3 \%$ for chromosome 6; 39.2 and $5.4 \%$ for chromosome 10; 40.7 and $4 \%$ for chromosome 17.

\section{Results}

\section{Clinical Findings}

All patients were male and the ages at presentation were 44 years (case 1), 47 years (case 2), and 60 years (case 3) (median age: 50 years). Primary kidney disease was related to hypertension in one patient (case 1), whereas the other two patients (cases 2 and 3 ) had chronic glomerulonephritis. A history of hemodialysis was reported for all three patients, with the duration of 96 months (case 1), 140 months (case 2), and 8 months (case 3), respectively, before the diagnosis of renal tumor. One patient (case 1) died of acute renal failure 34 months after surgery, whereas the other two patients (cases 2 and 3) were alive without evidence of disease 180 and 132 months after surgery, respectively.

\section{Pathologic Findings}

Case 1 showed a single $25 \mathrm{~mm}$ well-demarcated pseudo-encapsulated tumor. It was composed of
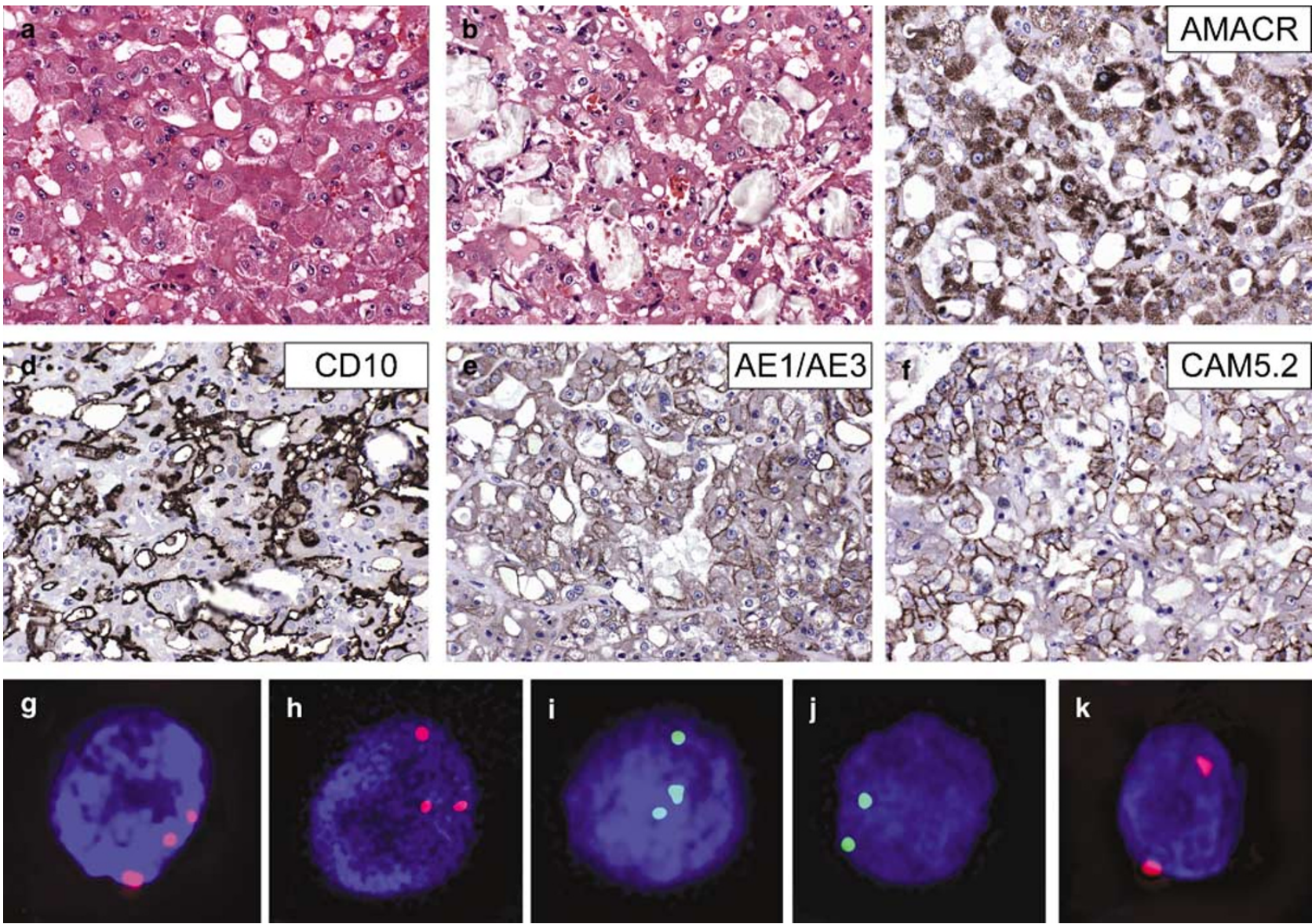

Figure 1 Case 1. (a) The tumor was composed of large, eosinophilic cells arranged in a solid pattern with cribriform appearance; (b) numerous oxalate crystals were appreciable within the tumor; (c) immunohistochemistry for AMACR showing strong, granular cytoplasmic staining; (d) immunohistochemistry for CD10 with luminal immunoreactivity, highlighting lumen-like spaces; (e) immunohistochemistry for AE1/AE3 showing membranous immunostaining; (f) immunohistochemistry for CAM5.2 showing membranous immunostaining. FISH analyses with centromeric probes showing nuclei with three hybridization signals for chromosome 1 (g), chromosome 2 (h), and chromosome 6 (i), consistent with chromosomal gains; nuclei with two hybridization signals were observed for chromosome 10 (j) and chromosome $17(\mathbf{k})$. 
cells with large vesicular nuclei, prominent nucleoli easily visible with the $\times 10$ objective, and abundant eosinophilic, focally coarsely granular, cytoplasm (Figure 1). Mitotic activity was nil. The tumor showed a solid pattern of growth. A cribriform appearance, due to the presence of numerous large, irregularly shaped intracytoplasmic vacuoles, was present in some areas. Numerous calcium oxalate crystals were present and the amount of oxalate deposition was grade $3+$, according to the method of a previous report. ${ }^{14}$ A few basophilic microcalcifications were present. The surrounding renal parenchyma was extensively replaced by cysts lined by cuboidal epithelium, which sometimes showed small foci of hyperplastic and papillary changes, typical of acquired cystic renal disease.

Case 2 showed a $15 \mathrm{~mm}$ tumor composed primarily of medium-sized cells with finely granular eosinophilic cytoplasm. In these areas, there were numerous large variably shaped intracytoplasmic vacuoles, which imparted a cribriform appearance.
There were also microscopic cysts, most of which were filled with hemorrhage. In the center of the tumor was an area with a more solid appearance in which the cells were larger and had cytoplasm and prominent plasma membranes reminiscent of classic chromophobe renal cell carcinoma. Throughout the tumor, the nuclei were round or slightly irregular, had nucleoli easily visible with the $\times 10$ objective, and lacked mitotic figures. Calcium oxalate crystals were sparse and were graded as $1+$. The surrounding renal parenchyma contained numerous cysts (Figure 2).

Case 3 showed an $8 \mathrm{~mm}$ well-circumscribed pseudo-encapsulated tumor with a tubulo-cystic growth pattern, composed of cells with eosinophilic cytoplasm and irregular nuclei, often with nucleoli easily visible with the $\times 10$ objective. Calcium oxalate deposits were also present and their amount was graded as $1+$. The remaining non-neoplastic parenchyma also displayed changes of acquired cystic kidney disease (Figure 3).
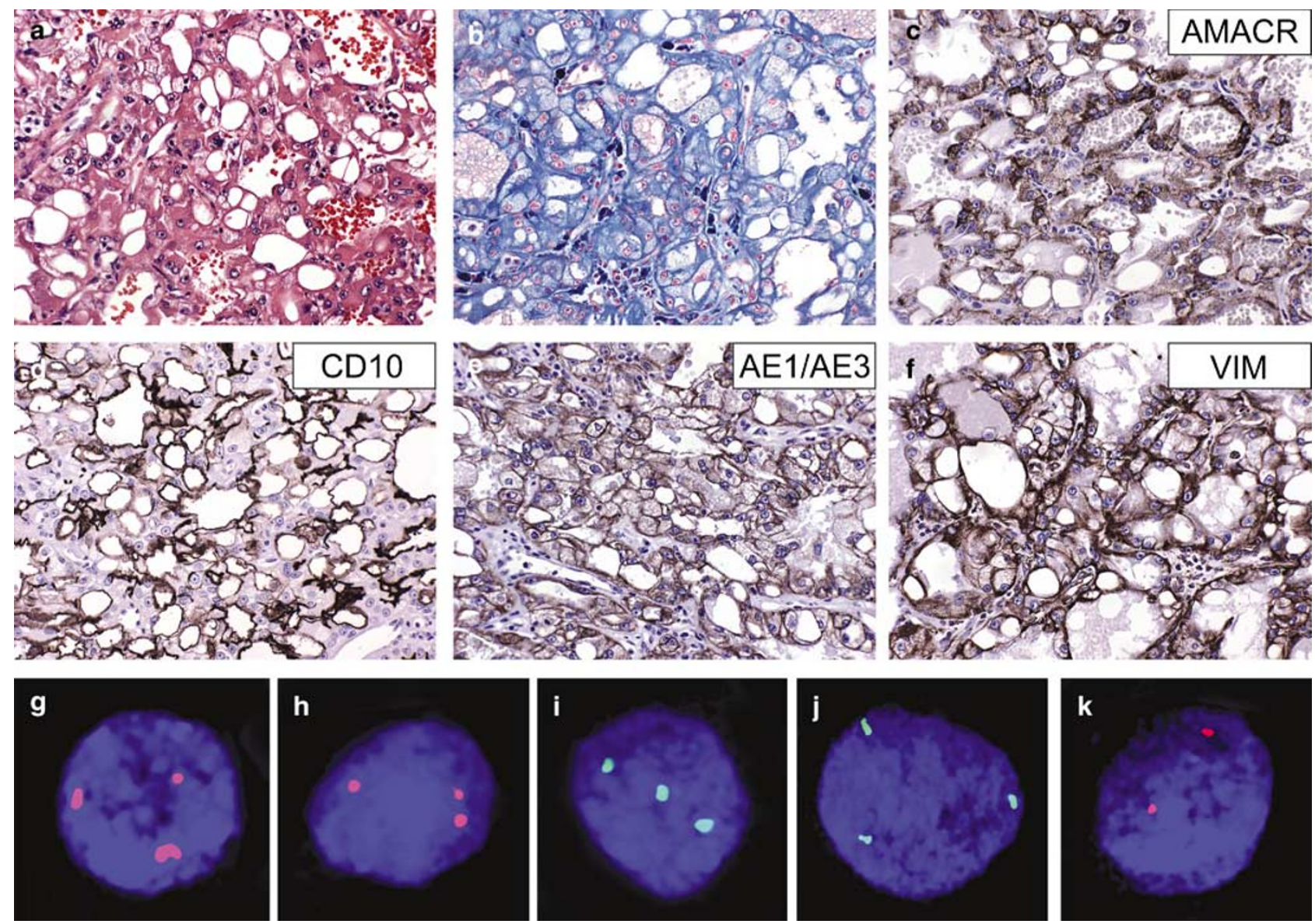

Figure 2 Case 2. (a) The tumor was composed mainly of large, eosinophilic cells with a focal component of clear cells, arranged in a solid or acinar architecture, with cribriform appearance; (b) Hale'stain showing a cytoplasmic positive reaction; (c) immunohistochemistry for AMACR displaying strong, granular cytoplasmic staining; (d) immunohistochemistry for CD10 with luminal immunoreactivity, highlighting lumen-like spaces; (e) immunohistochemistry for AE1/AE3 showing membranous immunostaining; (f) immunohistochemistry for vimentin showing cytoplasmic immunostaining. FISH analyses with centromeric probes showing nuclei with three hybridization signals for chromosome 1 (g), chromosome 2 (h), chromosome 6 (i), and chromosome 10 (j), consistent with chromosomal gains; nuclei with two hybridization signals were observed for chromosome 17 (k). 

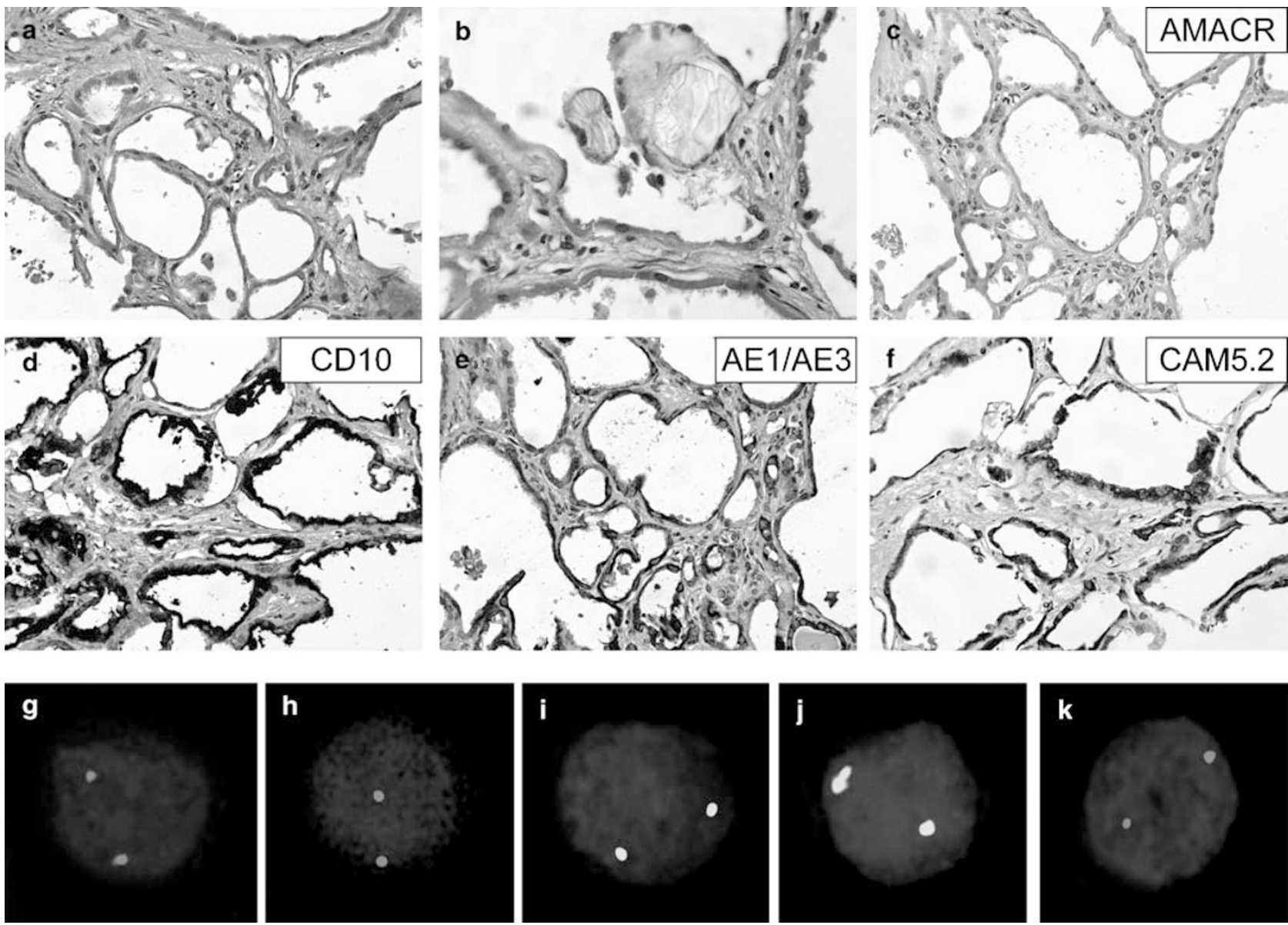

Figure 3 Case 3. (a) The tumor was characterized by the proliferation of eosinophilic cells in a microcystic pattern; (b) focal deposition of oxalate crystals was also observed; (c) immunohistochemistry with AMACR showed negative results; (d) immunohistochemistry for CD10 with luminal immunoreactivity; (e) immunohistochemistry for AE1/AE3 showing cytoplasmic and membranous immunostaining; (f) immunohistochemistry for CAM5.2 displaying mainly cytoplasmic immunostaining. FISH analyses with centromeric probes showing nuclei with two hybridization signals for chromosome 1 (g), chromosome 2 (h), chromosome 6 (i), chromosome 10 (j), and chromosome17 (k).

Table 1 Immunohistochemical results

\begin{tabular}{lcccccccc}
\hline Case \# & \multicolumn{9}{c}{ Antibodies } \\
\cline { 2 - 8 } & VIM & CAM5.2 & AE1/AE3 & EMA & CK7 & HMWK & CD10 & AMACR \\
\hline 1 & Neg & Pos & Pos & Neg & Neg & Neg & Pos & Pos \\
2 & Pos & Neg & Pos & Neg & Neg & Neg & Pos & Pos \\
3 & Neg & Pos & Pos & Neg & Neg & Neg & Neg \\
\hline
\end{tabular}

AMACR, alpha-methylacyl-CoA-racemase; CK7, cytokeratin 7; EMA, epithelial membrane antigen; HMWK, high molecular weight keratin; Neg, negative; Pos, positive; VIM: vimentin.

\section{Histochemical Findings}

Hale's colloidal iron stain was negative in cases 1 and 3 , whereas case 2 showed diffuse cytoplasmic staining ranging from moderate to strong.

\section{Immunohistochemistry}

The results of the immunohistochemical procedures are summarized in Table 1.
Vimentin was expressed in case 2 with moderate to strong intensity, and was not expressed in other cases. CAM5.2 was positive in both case 1 , mainly with membranous staining, and case 3 , with cytoplasmic and membranous staining, whereas case 2 was negative. AE1/AE3 was positive in all three cases with a membranous (cases 1 and 2) or cytoplasmic and membranous (case 3) immunoreactivity. CD10 showed a strong, diffuse immunostaining in all the tumors, mainly with a luminal distribu- 
Table 2 Fluorescence in situ hybridization results

\begin{tabular}{|c|c|c|c|c|c|c|}
\hline \multirow[t]{2}{*}{ Case \# } & \multicolumn{3}{|c|}{ Chromosome 1} & \multicolumn{3}{|c|}{ Chromosome 2} \\
\hline & $1 \operatorname{signal}(\%)$ & 2 signals (\%) & $\geq 3$ signals (\%) & $1 \operatorname{signal}(\%)$ & 2 signals (\%) & $\geq 3$ signals $(\%)$ \\
\hline 1 & 30.2 & 62.9 & 6.9 & 40.7 & 54.1 & 5.2 \\
\hline 2 & 38.0 & 55.7 & 6.3 & 32.9 & 59.9 & 7.2 \\
\hline 3 & 34.6 & 60.6 & 4.8 & 39.3 & 57.1 & 3.6 \\
\hline \multirow[t]{2}{*}{ Case \# } & \multicolumn{3}{|c|}{ Chromosome 6} & \multicolumn{3}{|c|}{ Chromosome 10} \\
\hline & 1 signal (\%) & 2 signals (\%) & $\geq 3$ signals (\%) & 1 signal (\%) & 2 signals (\%) & $\geq 3$ signals $(\%)$ \\
\hline 1 & 31.7 & 55.2 & 13.1 & 36.3 & 57.3 & 3.2 \\
\hline 2 & 35.0 & 54.1 & 10.9 & 27.9 & 62.8 & 9.3 \\
\hline 3 & 37.0 & 59.3 & 3.7 & 32.5 & 64.0 & 3.5 \\
\hline \multirow[t]{2}{*}{ Case \# } & \multicolumn{3}{|c|}{ Chromosome 17} & & & \\
\hline & 1 signal (\%) & 2 signals $(\%)$ & $\geq 3$ signals $(\%)$ & & & \\
\hline 1 & 37.8 & 58.4 & 3.8 & & & \\
\hline 2 & 31.2 & 65.2 & 3.6 & & & \\
\hline 3 & 36.9 & 59.6 & 3.5 & & & \\
\hline
\end{tabular}

tion, sometimes highlighting the lumen-like spaces appreciable in cases 1 and 2. AMACR was expressed in cases 1 and 2 with a strong and diffuse granular cytoplasmic immunostaining. EMA, CK7, and HMWK were not expressed in any of the tumors.

\section{Fluorescence In Situ Hybridization}

The results of the FISH analyses are summarized in Table 2. Using the criteria for chromosome gains (see Materials and methods section), case 1 had gains of chromosomes 1,2, and 6, whereas case 2 had gains of chromosomes 1, 2, 6, and 10. Cases 1 and 2 showed no loss of any of the chromosomes. No chromosomal losses or gains were observed in case 3 (Figure 1).

\section{Discussion}

We analyzed three unusual eosinophilic renal epithelial tumors occurring in kidneys with acquired cystic disease. These tumors were composed of primarily of large cells with finely granular eosinophlic cytoplasm arranged in solid, acinar, or microcystic patterns. Two of the tumors had a distinctive cribriform appearance caused by numerous irregularly shaped vacuoles. Each tumor contained deposits of calcium oxalate crystals. Our study found that these tumors have immunophenotypes and cytogenetic findings that differ from those of the common types of renal cell neoplasia.

Although end-stage renal disease-associated and acquired renal cystic disease-associated renal tumors with extensive components of eosinophilic cells have been previously reported,,$^{11,12,26,27}$ the recognition of some of these tumors as distinctive clinicopathologic entities is a recent advance. Tickoo et $a l^{13}$ described in abstract form the wide spectrum of epithelial tumors occurring in end-stage renal disease, identifying a peculiar histological pattern in 15 out of 32 tumors associated with acquired cystic kidney disease. The dominant morphologic features in these tumors, for which Tickoo et $a l^{13}$ proposed the name 'acquired cystic kidney disease-specific renal cell carcinomas', included solid, cribriform, or papillary architecture, composed mainly of eosinophilic cells (sometimes with a small component of cells with pale or clear cytoplasm) and calcium oxalate crystal deposition within the tumors. Recently, Sule et $a l^{14}$ reported a series of seven tumors characterized by variable deposition of calcium oxalate crystals in five patients with acquired cystic disease, identifying them as neoplasms with 'oxalate' phenotype, which shared distinctive cytologic and histologic features including eosinophilic cytoplasm, large nuclei with prominent nucleoli, and variable patterns of growth including papillary, tubulocystic, solid, and cribriform. The tumors we investigated share morphologic similarities with the cases previously described in association with acquired cystic disease, including the presence of calcium oxalate crystals. Calcium oxalate crystals may be a unique feature of these tumors, as they have not been observed in sporadic renal epithelial tumors. ${ }^{11,13,14,28}$ The role of the calcium oxalate crystals in the development of renal cell tumors in the context of acquired cystic disease of kidney is uncertain. ${ }^{11,14,29}$

Immunohistochemical studies on acquired cystic disease-associated renal tumors are few, and mostly 
limited to the common types: clear-cell renal carcinomas and papillary renal cell carcinomas. ${ }^{7,8}$ Sule et $a l^{14}$ analyzed seven eosinophilic epithelial tumors with intratumoral oxalate crystals, associated with acquired cystic kidney disease, with antibodies for RCC marker, CD10, epithelial membrane antigen, high molecular weight cytokeratin, and a kidneyspecific cadherin, demonstrating that all the neoplasms shared a similar immunophenotype, namely strong reactions for RCC marker and CD10, whereas the reactions for EMA, HMWK, and kidney-specific cadherin were weak and focal. In our study, we performed immunohistochemical analyses with antibodies against cytokeratins (CAM5.2, AE1/AE3, EMA, CK7, and HMWK), vimentin, CD10, and AMACR. We found that all tumors had diffuse immunoreactivity for AE1/AE3 and CD10, whereas AMACR was strongly positive only in tumors with solid or acinar architecture and cribriform features (cases 1 and 2). Two tumors (cases 1 and 3) also showed positive reactions for CAM5.2, whereas vimentin was positive only in one case (case 2). EMA, CK7, and HMWK were negative in all three tumors. One tumor (case 2) also showed strong Hale's colloidal iron staining.

Genetic analyses of acquired cystic disease-associated renal tumors are scarce, and almost entirely have been studies of the common renal cell carcinomas. ${ }^{5,12,26,27,30-32}$ Tickoo et $a l^{13}$ reported FISH analyses for von Hippel Lindau ( $V H L$ ) gene and chromosomes 7 and 17 on 43 tumors arising in patients with end-stage renal disease, among which 15 occurred in kidneys with acquired renal cystic disease. The authors found that the tumors in kidneys with acquired cystic kidney disease had no VHL gene deletions, whereas the gains of chromosomes 7 and 17 were observed. Recently, O'Reilly et $a{ }^{33}$ described in abstract form FISH analyses with centromeric probes for chromosomes 1, 3, 7, and 17 on 10 tumors arising in end-stage renal disease, among which two acquired cystic kidney diseaseassociated tumors were included. They found that both tumors were disomic for chromosomes 1 and 3, whereas one had loss of chromosome 7 and the other showed loss of chromosome 17. Our FISH analysis demonstrated that the three tumors included in this study did not show any chromosomal losses for chromosomes $1,2,6,10$, or 17 , whereas the two tumors with solid growth patterns punctuated by vacuoles giving a cribriform appearance had gains of multiple chromosomes: chromosomes 1, 2, and 6 in case 1 and chromosomes 1, 2, 6, and 10 in case 2 . Our data suggest that these tumors have a distinctive genetic profile, and show that these tumors do not have the multiple chromosomal losses that are typical of chromophobe renal cell carcinomas, nor the loss of chromosome 1 found in approximately $10 \%$ of renal oncocytomas. ${ }^{16-20}$ As the differential diagnosis of renal epithelial tumors with abundant eosinophilic cytoplasm includes the eosinophilic variant of chromophobe renal cell carcinoma and renal oncocytoma, FISH analyses could contribute to rendering an accurate diagnosis of histologically challenging cases in this setting.

In conclusion, our study demonstrated that acquired cystic disease-associated eosinophilic renal tumors show peculiar immunophenotypes that differ from those of the common renal cell neoplasms. Our FISH analyses demonstrated that these tumors did not show any of the chromosomal losses that are typical of the eosinophilic variants of chromophobe renal cell carcinomas. Gains of chromosomes 1, 2, and 6 were present in two of the three tumors. Our data support the concept that these tumors are a distinctive clinicopathologic entity in the spectrum of renal cell neoplasms.

\section{References}

1 Denton MD, Magee CC, Ovuworie C, et al. Prevalence of renal cell carcinoma in patients with ESRD pretransplantation: a pathologic analysis. Kidney Int 2002;61:2201-2209.

2 Truong LD, Krishnan B, Cao JT, et al. Renal neoplasm in acquired cystic kidney disease. Am J Kidney Dis 1995;26:1-12.

3 Truong LD, Choi YJ, Shen SS, et al. Renal cystic neoplasms and renal neoplasms associated with cystic renal diseases: pathogenetic and molecular links. Adv Anat Pathol 2003;10:135-159.

4 Ishikawa I, Kovacs G. High incidence of papillary renal cell tumours in patients on chronic haemodialysis. Histopathology 1993;22:135-139.

5 Gronwald J, Baur AS, Holtgreve-Grez H, et al. Chromosomal abnormalities in renal cell neoplasms associated with acquired renal cystic disease. A series studied by comparative genomic hybridization and fluorescence in situ hybridization. J Pathol 1999;187:308-312.

6 Hughson MD, Buchwald D, Fox M. Renal neoplasia and acquired cystic kidney disease in patients receiving long-term dialysis. Arch Pathol Lab Med 1986;110: 592-601.

7 Ikeda R, Tanaka T, Moriyama MT, et al. Proliferative activity of renal cell carcinoma associated with acquired cystic disease of the kidney: comparison with typical renal cell carcinoma. Hum Pathol 2002;33:230-235.

8 Khurana KK, Truong LD, Verani RR. Image analysis of proliferating cell nuclear antigen expression and immunohistochemical profiles in renal cell carcinoma associated with acquired cystic kidney disease: comparison with classic renal cell carcinoma. Mod Pathol 1998;11:339-346.

9 Konda R, Sato H, Hatafuku F, et al. Expression of hepatocyte growth factor and its receptor C-met in acquired renal cystic disease associated with renal cell carcinoma. J Urol 2004;171:2166-2170.

10 Peces R, Martinez-Ara J, Miguel JL, et al. Renal cell carcinoma co-existent with other renal disease: clinico-pathological features in pre-dialysis patients and those receiving dialysis or renal transplantation. Nephrol Dial Transplant 2004;19:2789-2796.

11 Rioux-Leclercq NC, Epstein JI. Renal cell carcinoma with intratumoral calcium oxalate crystal deposition in patients with acquired cystic disease of the kidney. Arch Pathol Lab Med 2003;127:E89-E92. 
12 Hughson MD, Bigler S, Dickman K, et al. Renal cell carcinoma of end-stage renal disease: an analysis of chromosome 3, 7, and 17 abnormalities by microsatellite amplification. Mod Pathol 1999;12:301-309.

13 Tickoo SK, de Peralta-Venturina MN, Salama M, et al. Spectrum of epithelial tumors in end stage renal disease (ESRD): emphasis on histologic patterns distinct from those in sporadic adult renal neoplasia. Lab Invest 2003;83:173A.

14 Sule N, Yakupoglu U, Shen SS, et al. Calcium oxalate deposition in renal cell carcinoma associated with acquired cystic kidney disease: a comprehensive study. Am J Surg Pathol 2005;29:443-451.

15 Eble JN, Sauter G, Epstein JI, et al. Tumours of the Kidney World Health Organization Classification of Tumours: Pathology and Genetics of Tumours of the Urinary System and Male Genital Organs. IARC Press: Lyon, 2004.

16 Brunelli M, Eble JN, Zhang S, et al. Eosinophilic and classic chromophobe renal cell carcinomas have similar frequent losses of multiple chromosomes from among chromosomes $1,2,6,10$, and 17 , and this pattern of genetic abnormality is not present in renal oncocytoma. Mod Pathol 2005;18:161-169.

17 Kuroda N, Toi M, Hiroi M, et al. Review of chromophobe renal cell carcinoma with focus on clinical and pathobiological aspects. Histol Histopathol 2003;18: 165-171.

18 Kuroda N, Toi M, Hiroi M, et al. Review of renal oncocytoma with focus on clinical and pathobiological aspects. Histol Histopathol 2003;18:935-942.

19 Schwerdtle RF, Storkel S, Neuhaus C, et al. Allelic losses at chromosomes 1p, 2p, 6p, 10p, 13q, 17p, and $21 q$ significantly correlate with the chromophobe subtype of renal cell carcinoma. Cancer Res 1996;56: 2927-2930.

20 Fuzesi L, Frank D, Nguyen C, et al. Losses of 1p and chromosome 14 in renal oncocytomas. Cancer Genet Cytogenet 2005;160:120-125.

21 Cossu-Rocca P, Zhang S, Roth LM, et al. Chromosome $12 \mathrm{p}$ abnormalities in dysgerminoma of the ovary: a FISH analysis. Mod Pathol 2006;19:611-615.

22 Cossu-Rocca P, Eble JN, Delahunt B, et al. Renal mucinous tubular and spindle carcinoma lacks the gains of chromosomes 7 and 17 and losses of chromosome $\mathrm{Y}$ that are prevalent in papillary renal cell carcinoma. Mod Pathol 2006;19:488-493.
23 Kernek KM, Brunelli M, Ulbright TM, et al. Fluorescence in situ hybridization analysis of chromosome $12 \mathrm{p}$ in paraffin-embedded tissue is useful for establishing germ cell origin of metastatic tumors. Mod Pathol 2004;17:1309-1313.

24 Brunelli M, Eble JN, Zhang S, et al. Metanephric adenoma lacks the gains of chromosomes 7 and 17 and loss of $\mathrm{Y}$ that are typical of papillary renal cell carcinoma and papillary adenoma. Mod Pathol 2003;16: 1060-1063.

25 Brunelli M, Eble JN, Zhang S, et al. Gains of chromosomes 7, 17, 12, 16, and 20 and loss of Y occur early in the evolution of papillary renal cell neoplasia: a fluorescent in situ hybridization study. Mod Pathol 2003;16:1053-1059.

26 Hughson MD, Meloni AM, Silva FG, et al. Renal cell carcinoma in an end-stage kidney of a patient with a functional transplant: cytogenetic and molecular genetic findings. Cancer Genet Cytogenet 1996;89:65-68.

27 Hughson MD, Schmidt L, Zbar B, et al. Renal cell carcinoma of end-stage renal disease: a histopathologic and molecular genetic study. J Am Soc Nephrol 1996;7: 2461-2468.

28 Dry SM, Renshaw AA. Extensive calcium oxalate crystal deposition in papillary renal cell carcinoma: report of two cases. Arch Pathol Lab Med 1998;122: 260-261.

29 Sant GR, Ucci Jr AA. Acquired renal cystic disease and adenocarcinoma following renal transplantationa current urologic perspective. Urol Int 1998;60: 108-112.

30 Cheuk W, Lo ES, Chan AK, et al. Atypical epithelial proliferations in acquired renal cystic disease harbor cytogenetic aberrations. Hum Pathol 2002;33:761-765

31 Yoshida M, Yao M, Ishikawa I, et al. Somatic von Hippel-Lindau disease gene mutation in clear-cell renal carcinomas associated with end-stage renal disease/acquired cystic disease of the kidney. Genes Chromosomes Cancer 2002;35:359-364.

32 Ishikawa I, Shikura N, Ozaki M. Papillary renal cell carcinoma with numeric changes of chromosomes in a long-term hemodialysis patient: a karyotype analysis. Am J Kidney Dis 1993;21:553-556.

33 O’Reilly KC, Tickoo SK, Amin MB, et al. Renal cell tumors arising in end stage renal disease (ESRD): a karyotypic study with fluorescent-in-situ-hybridization (FISH). Mod Pathol 2005;18:156A. 